\title{
A new Hydrocephalus Clinical Research Network protocol to reduce cerebrospinal fluid shunt infection
}

\author{
John R. W. Kestle, MD, ${ }^{1}$ Richard Holubkov, PhD, ${ }^{1}$ D. Douglas Cochrane, MD, ${ }^{2}$ \\ Abhaya V. Kulkarni, MD, ${ }^{3}$ David D. Limbrick Jr., MD, PhD, ${ }^{4}$ Thomas G. Luerssen, MD, ${ }^{5}$ \\ W. Jerry Oakes, MD, ${ }^{6}$ Jay Riva-Cambrin, MD, MSc, ${ }^{1}$ Curtis Rozzelle, MD, ${ }^{6}$ \\ Tamara D. Simon, MD, MSPH, ${ }^{7}$ Marion L. Walker, MD, ${ }^{1}$ John C. Wellons III, MD, MSPH, ${ }^{8}$ \\ Samuel R. Browd, MD, PhD, ${ }^{7}$ James M. Drake, MBBCh, ${ }^{3}$ Chevis N. Shannon, MBA, MPH, DrPH, ${ }^{8}$ \\ Mandeep S. Tamber, MD, PhD, ${ }^{9}$ and William E. Whitehead, MD, MPH, ${ }^{5}$ for the Hydrocephalus \\ Clinical Research Network
}

\begin{abstract}
${ }^{1}$ Department of Neurosurgery, University of Utah, Salt Lake City, Utah; ${ }^{2}$ Division of Pediatric Neurosurgery, British Columbia Children's Hospital, Vancouver, British Columbia, ${ }^{3}$ Division of Neurosurgery, Hospital for Sick Children, Toronto, Ontario, Canada; ${ }^{4}$ Department of Neurosurgery, St. Louis Children's Hospital, St. Louis, Missouri; ${ }^{5}$ Department of Neurosurgery, Texas Children's Hospital, Houston, Texas; ' Section of Pediatric Neurosurgery, Children's Hospital of Alabama, Birmingham, Alabama; ${ }^{7}$ Department of Pediatrics, Division of Hospital Medicine, Seattle Children's Hospital, Seattle, Washington; ${ }^{8}$ Department of Neurosurgery, Monroe Carell Jr. Children's Hospital at Vanderbilt, Nashville, Tennessee; and 'Department of Neurosurgery, Children's Hospital of Pittsburgh of UPMC, Pittsburgh, Pennsylvania
\end{abstract}

OBJECTIVE In a previous report by the same research group (Kestle et al., 2011), compliance with an 11-step protocol was shown to reduce CSF shunt infection at Hydrocephalus Clinical Research Network (HCRN) centers (from 8.7\% to 5.7\%). Antibiotic-impregnated catheters (AICs) were not part of the protocol but were used off protocol by some surgeons. The authors therefore began using a new protocol that included AICs in an effort to reduce the infection rate further.

METHODS The new protocol was implemented at HCRN centers on January 1, 2012, for all shunt procedures (excluding external ventricular drains [EVDs], ventricular reservoirs, and subgaleal shunts). Procedures performed up to September 30, 2013, were included (21 months). Compliance with the protocol and outcome events up to March 30, 2014, were recorded. The definition of infection was unchanged from the authors' previous report.

RESULTS A total of 1935 procedures were performed on 1670 patients at 8 HCRN centers. The overall infection rate was $6.0 \%$ (95\% Cl 5.1\%-7.2\%). Procedure-specific infection rates varied (insertion 5.0\%, revision 5.4\%, insertion after EVD $8.3 \%$, and insertion after treatment of infection 12.6\%). Full compliance with the protocol occurred in $77 \%$ of procedures. The infection rate was $5.0 \%$ after compliant procedures and $8.7 \%$ after noncompliant procedures $(p=0.005)$. The infection rate when using this new protocol $(6.0 \%, 95 \% \mathrm{Cl} 5.1 \%-7.2 \%)$ was similar to the infection rate observed using the authors' old protocol $(5.7 \%, 95 \% \mathrm{Cl} 4.6 \%-7.0 \%)$.

CONCLUSIONS CSF shunt procedures performed in compliance with a new infection prevention protocol at HCRN centers had a lower infection rate than noncompliant procedures. Implementation of the new protocol (including AICs) was associated with a $6.0 \%$ infection rate, similar to the infection rate of $5.7 \%$ from the authors' previously reported protocol. Based on the current data, the role of AICs compared with other infection prevention measures is unclear.

http://thejns.org/doi/abs/10.3171/2015.8.PEDS15253

KEY WORDS cerebrospinal fluid; shunt infection; quality improvement; protocol; hydrocephalus

ABBREVIATIONS AIC = antibiotic-impregnated catheter; EVD = external ventricular drain; HCRN = Hydrocephalus Clinical Research Network. SUBMITTED April 27, 2015. ACCEPTED August 24, 2015.

INCLUDE WHEN CITING Published online December 18, 2015; DOI: 10.3171/2015.8.PEDS15253. 
I NFECTION continues to be a common complication of CSF shunts for children with hydrocephalus, and there are ongoing efforts to identify methods or devices that may reduce this risk. Quality-improvement research has suggested that standardized protocols may reduce device-related infection in a number of areas., ${ }^{1,3,11}$ The Hydrocephalus Clinical Research Network (HCRN) has used this approach to minimize shunt infection rates since 2007. A protocol was developed using the available literature that included 11 steps aimed at reducing infection, such as provider hand washing and double gloving, use of perioperative antibiotics, and patient positioning. An assessment of this protocol in 2011 demonstrated a reduced infection rate compared with baseline. ${ }^{7}$

Since that report, the HCRN centers have continued to use a standard protocol and record compliance; however, as antibiotic-impregnated shunt catheters (AICs) became more popular, some surgeons began using them in addition to the protocol. This prompted us to develop a new protocol that included AICs. The purpose of this report is to evaluate whether the new protocol was associated with a further reduction in the shunt infection rate.

\section{Methods}

The HCRN is a collaborative group of pediatric neurosurgical centers conducting clinical research in pediatric hydrocephalus. At present, there are 9 centers and 17 investigators (listed in Appendix) contributing data to HCRN studies. From its inception, the HCRN has used quality-improvement methodology in an effort to reduce shunt infection.

\section{Protocol Refinement}

The 11-step protocol applied from June 1, 2007, to February 28, 2009, was continued through December 31, 2011. As part of our ongoing quality-improvement process, we analyzed compliance and events on the protocol through March 31, 2011, to consider revisions to it. This revealed increasing use of AICs in addition to the 11 steps of the protocol; AICs were used in 344 of 1057 procedures at two centers. We therefore decided to adopt a new protocol that includes AICs and assess their impact on the infection rate. To simplify the protocol we removed several other steps, including the injection of vancomycin and gentamicin into the shunt, the orientation of the surgical field with respect to the operating room door, the sign on the door restricting operating room traffic, and the requirement for hair clipping rather than shaving. The new protocol, as outlined in Fig. 1, was implemented at the HCRN centers from January 1, 2012, through September 30, 2013. Patients were monitored for 6 months after shunt insertion or revision, and subsequent surgical and/or infectious events were recorded.

\section{Study Sample}

The entry criteria and outcome criteria were identical to those used in the previous publication. ${ }^{7}$ All children at each HCRN center were entered into the study when they underwent a shunt insertion or shunt revision operation, including ventriculoperitoneal, ventriculoatrial, ventricu-

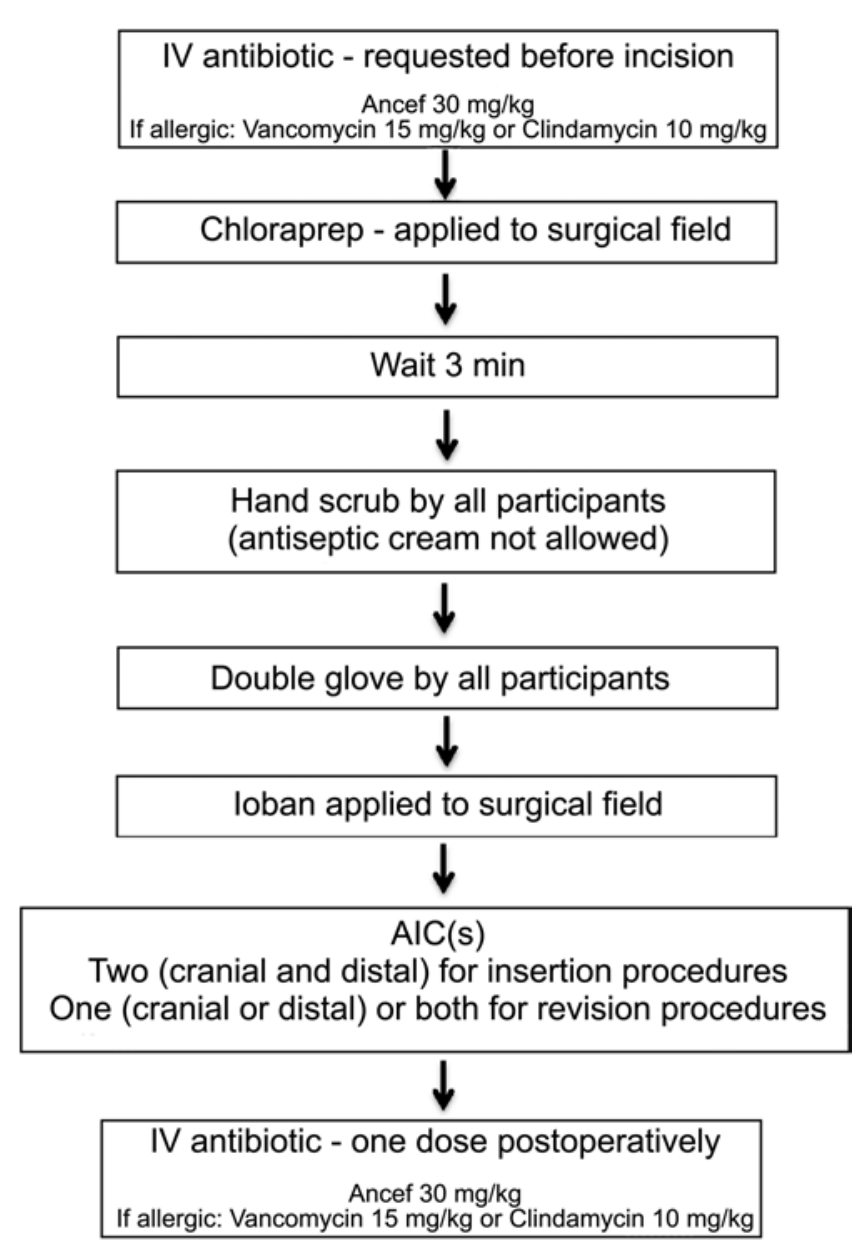

FIG. 1. New HCRN protocol to reduce shunt infection.

lopleural, arachnoid cyst, and subdural-peritoneal shunts and shunts inserted after treatment of an infection. Patients whose first presentation to the study cohort was with a shunt infection were entered into the study at the time their shunts were being replaced after treatment of their infections. Children undergoing placement of an external ventricular drain (EVD), Ommaya reservoir, ventricular access device, or subgaleal shunt were not included at the time of those procedures but were eligible if they later underwent a shunt procedure listed above.

Patients were evaluated for infection at the time of routine clinical follow-up, emergency room visits, or hospital admission. Evaluation followed the surgeons' usual clinical practice. The primary end point for the study was shunt infection, defined as follows: 1) identification of organisms on culture or Gram stain from CSF, wound swab, or pseudocyst fluid; 2) shunt erosion (defined as wound breakdown with visible shunt hardware); 3) abdominal pseudocyst (even in the absence of positive cultures); or 4) positive blood cultures in a child with a ventriculoatrial shunt.

Procedures were classified as shunt insertion (insertion of a shunt in a child who had not had one previously), shunt revision (surgery in which a child entered the operating room with all shunt equipment previously implanted and left the operating room with all shunt equipment 
implanted-i.e., they had no externalized components or drains before or after surgery), shunt insertion after external ventricular drainage (not infected), and shunt insertion after treatment of infection. All repeat procedures of any type were recorded.

Compliance with protocol steps was assessed prospectively by HCRN coordinators, surgeons, fellows, and residents. In addition, several surgeon-specific nonprotocol steps that might reduce infection were recorded. These included changing the outer gloves after draping, using a "no-touch" operative technique in which the shunt equipment is only handled with surgical instruments, and irrigating wounds with Bacitracin solution before closure. Data were retained at each center until institutional review board approval was obtained for data pooling and analysis. Data from 1 of the 9 centers did not include procedures done in the evenings or on weekends. That center provided data on 65 shunt procedures, but without the evening or weekend events we felt that important outcomes might be missing. The analysis was therefore performed using 8 of 9 HCRN centers. A "compliant" procedure was one in which all protocol steps were performed. For shunt insertion procedures (including initial shunt insertion, shunt insertion after EVD placement, and shunt insertion after infection), "compliance" meant that the ventricular catheter and the peritoneal catheter were both AICs (in addition to all the other protocol steps). A shunt revision procedure was considered compliant if all steps of the protocol were followed and the ventricular catheter or the peritoneal catheter (or both) was an AIC. The commercial brand of the AIC was left up to the surgeon.

\section{Analysis}

Categorical outcomes were compared with a Pearson chi-square test or Fisher exact test of proportions. Logistic regression was performed to assess for associations between protocol or nonprotocol factors and infection. The regression models adjusted for within-patient correlation (as often the same patient was seen multiple times during the period of this study) using generalized estimating equations. One model included only components of the shunt protocol, whereas a second model tested the association of the protocol as well as additional nonprotocol factors with infection. Analysis was performed using commercially available statistical software (SPSS version 17.0, SAS version 9.2).

\section{Results}

There were 1935 procedures performed on 1670 pa-
TABLE 2. Center-specific volume and infection rates

\begin{tabular}{ccc}
\hline Center No. & Center Vol & ${\text { Center Infection Rate }(\%)^{*}}^{*}$ \\
\hline 1 & 457 & 8.3 \\
\hline 2 & 377 & 5.8 \\
\hline 3 & 319 & 3.8 \\
\hline 4 & 314 & 6.1 \\
\hline 5 & 291 & 5.8 \\
\hline 6 & 150 & 5.3 \\
\hline 8 & 16 & 6.3 \\
\hline
\end{tabular}

${ }^{*} p=0.34$.

tients at 8 HCRN centers from January 1, 2012, through September 30, 2013. Repeat procedures were captured for 6 months after each patient was entered into the study (through March 31, 2014). The most common procedure was shunt revision, which accounted for 1193 (61.7\%) of the 1935 procedures, followed by shunt insertion (482 procedures, $24.9 \%$, shunt insertion after infection (151 procedures, $7.8 \%$ ), and shunt insertion after EVD without infection (109 procedures, $5.6 \%$ ).

The overall infection rate was $6.0 \%$ (117/1935 procedures), and infection rates varied by procedure ( $\mathrm{p}=0.002$, Table 1), with the highest infection rate of $12.6 \%$ for shunt insertion after treatment of an infection. The infection rate at 7 of the 8 centers ranged from $3.8 \%$ to $8.3 \%$ and was not obviously associated with center volume (Table 2). One of the centers that had recently joined HCRN entered 11 patients and had no infections.

Compliance with the protocol steps was recorded. There were 185 procedures with incomplete compliance data. Among the remaining 1751 procedures, 1349 (77\%) were fully compliant with the protocol. The infection rate for fully compliant procedures was $5.0 \%$ and that for procedures that were not fully compliant was $8.7 \%$ ( $\mathrm{p}=$ 0.005 ). Compliance appeared to improve minimally over time (Fig. 2). Compliance was highest with shunt insertion procedures (89\%) and lowest with shunt insertion after infection procedures $(61 \%)$ (Table 1).

To assess the association of protocol and nonprotocol factors with infection, univariate comparisons and multivariate models were used. The univariate results (Table 3 ) suggested that preoperative antibiotics and AICs were associated with a lower infection rate.

Two logistic regression models were constructed using procedures with complete compliance data in all fields. In the model including all protocol factors (Table 3 ), the only

TABLE 1. Procedure-specific infection and compliance rates

\begin{tabular}{lccc}
\hline \multicolumn{1}{c}{ Shunt Procedure } & No. of Procedures & No. of Infections (\%) & \% Compliant Procedures \\
\hline Insertion & 482 & $24(5.0)$ & 89 \\
\hline Revision & 1193 & $64(5.4)$ & 75 \\
\hline Insertion after EVD (not infected) & 109 & $9(8.3)$ & 68 \\
\hline Insertion after infection & 151 & $19(12.6)$ & 61 \\
\hline Total & 1935 & $116(6.0)$ & 77 \\
\hline${ }^{*} p=0.002$. & & &
\end{tabular}




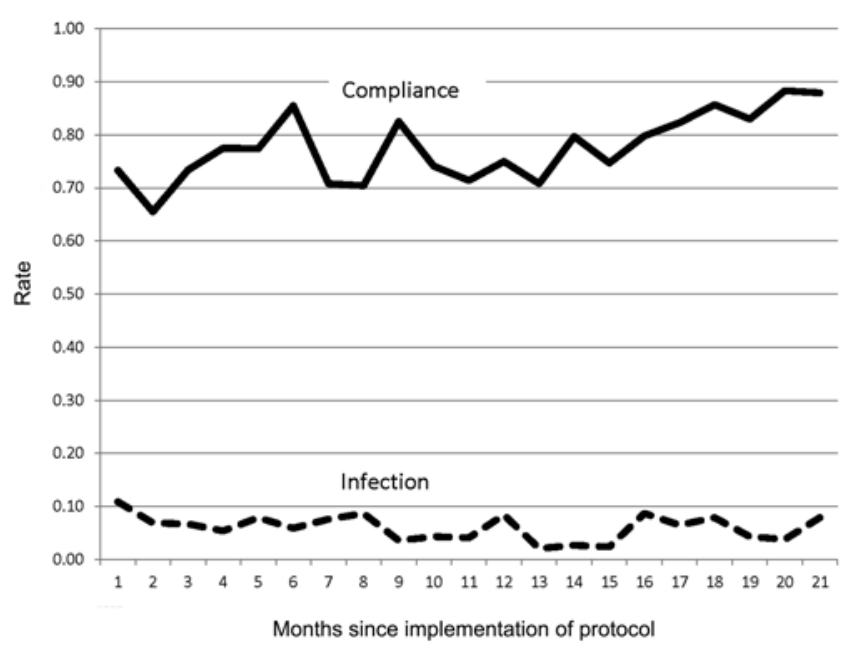

FIG. 2. Network-wide compliance and infection rates since implementation of the new protocol.

factor that was independently associated with infection was compliance with AICs (OR 1.9, 95\% CI 1.2-3.0, $\mathrm{p}=$ $0.0033)$. A second model included all protocol factors and the three additional nonprotocol factors (Table 3). Again, the only significant factor was compliance with AICs (OR $2.1,95 \%$ CI $1.3-3.4, \mathrm{p}=0.0026$ ).

The results of this protocol were compared with the results from our old protocol reported in 2011 (Fig. 3). ${ }^{7}$ The two protocols had similar compliance rates (old protocol $74.5 \%$, new protocol $77 \%$ ) The overall pooled infection rate from the 8 centers using the new protocol (including AICs) was $6.0 \%$ (95\% CI 5.1\%-7.2\%). The overall pooled infection rate from the old protocol ${ }^{7}$ with intrathecal antibiotics (not AICs) was 5.7\% (95\% CI 4.6\%-7.0\%). The HCRN has expanded from 4 to 9 centers since the publication of our old protocol. To improve the validity of the comparison of our current infection rate with the earlier infection rate, we restricted our analysis to the same 4 centers that were the basis for the 2011 report. When we did this, the infection rate on our new protocol was $6.7 \%(95 \%$
CI $5.4 \%-8.2 \%$ ), which appears to be similar to the infection rate we achieved with the old protocol.

\section{Discussion}

To minimize the risk of infection, we followed a standard protocol for shunt surgery at the HCRN centers. The infection rate was lower for compliant operations than for noncompliant operations, but the infection rate was not lower than we achieved with a different protocol in 2011.7

The use of standardized protocols to reduce infection in pediatric neurosurgery is not new. A number of singlecenter studies have demonstrated the ability to implement a standardized protocol and reduce infection. In 1992, Choux et al. ${ }^{2}$ reported a protocol for shunt implantation that was used from 1983 to 1990 . It included a series of steps in the preoperative, intraoperative, and postoperative periods. The infection rate for 1197 procedures (600 children) was $0.17 \%$, compared with $7.75 \%$ for 606 operations prior to the protocol (1978-1982). A no-touch technique protocol was reported in which the surgeons' hands did not touch the shunt equipment. In addition, traffic in the operating room was limited, educated assistants were used, patient preparation steps were followed, the operative field had two drapes, and surgeons wore double gloves. This approach was used in 60 procedures and reduced shunt infection to $2.9 \%$ from $9.1 \%(\mathrm{p}=0.058)$ seen in a previous group of 120 procedures. ${ }^{5}$ Another protocol including antibiotic prophylaxis, meticulous surgical technique, and complete shunt revision reduced the infection rate from $9.4 \%$ (382 operations) to $5.3 \%$ (112 operations). ${ }^{10}$ In 2007 , Pirotte et al. ${ }^{9}$ reported no infections for 115 procedures performed using a strict protocol of limited implant and skin-edge manipulation, minimal personnel in the operating room, shunt surgery first in the morning, avoiding CSF leak, surgery duration less than 30 minutes, and systemic antibiotic prophylaxis. They recorded compliance and found "errors in protocol application" in 71 of 115 procedures, which decreased with time. In a 2013 study of 1404 procedures, Hommelstad et al. ${ }^{6}$ implemented a perioperative protocol and reduced their infection rate from $6.5 \%$

TABLE 3. Compliance with protocol and nonprotocol factors

\begin{tabular}{|c|c|c|c|c|c|}
\hline \multirow[b]{2}{*}{ Factor } & \multirow{2}{*}{$\begin{array}{c}\text { Compliance } \\
\text { Rate (\%) }\end{array}$} & \multicolumn{2}{|c|}{ Infection Rate $(\%)$} & \multirow{2}{*}{$\begin{array}{c}p \\
\text { Value }\end{array}$} & \multirow{2}{*}{$\begin{array}{c}\text { No. of Ops } \\
\text { w/ Data } \\
\text { Recorded }\end{array}$} \\
\hline & & Compliant Ops & Noncompliant Ops & & \\
\hline \multicolumn{6}{|l|}{ Protocol factors } \\
\hline Intravenous antibiotics requested before incision & 99 & 5.9 & 17.6 & 0.08 & 1822 \\
\hline Chloraprep on surgical field & 99 & 5.8 & 13.6 & 0.29 & 1818 \\
\hline Wait 3 mins for Chloraprep to dry & 99 & 5.8 & 16.7 & 0.71 & 1775 \\
\hline All team members performed formal surgical scrub (no antiseptic cream) & 99 & 6.0 & 0 & 0.51 & 1818 \\
\hline All team members wore double gloves & 98 & 6.0 & 0 & 0.39 & 1817 \\
\hline Loban on surgical field & 99 & 5.9 & 16.7 & 0.50 & 1813 \\
\hline AICs used per protocol & 78 & 5.0 & 9.1 & 0.004 & 1857 \\
\hline \multicolumn{6}{|l|}{ Additional nonprotocol factors } \\
\hline Outer gloves changed after draping & 58 & 6.0 & 6.0 & 0.97 & 1774 \\
\hline No-touch surgical technique & 27 & 8.0 & 6.0 & 0.21 & 1382 \\
\hline Bacitracin irrigation of wounds & 92 & 5.8 & 8.9 & 0.35 & 1792 \\
\hline
\end{tabular}




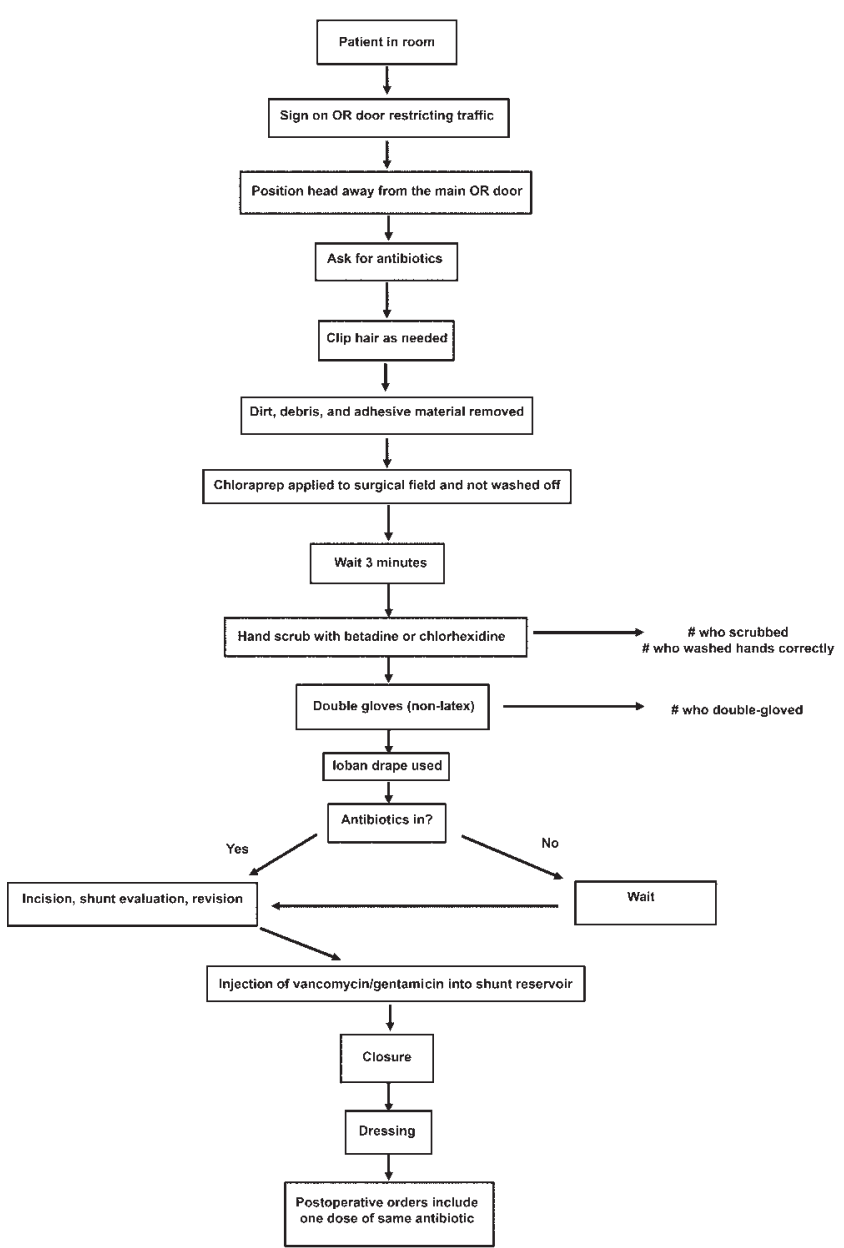

FIG. 3. Old HCRN protocol from 2011.

to $4.3 \%$ overall and $18.4 \%$ to $5.7 \%$ for procedures in children less than 1 year of age. These studies have achieved a range of infection rates, some of which were lower than our 6.0\%; however, they were all single-center studies that would likely have had reduced variation in practice compared with our multicenter network. In addition, they had various definitions of infection (some of which were not too precise), making comparisons with our results difficult.

AICs have been studied extensively in the last decade. They have been the subject of a recent meta-analysis suggesting a reduced infection rate with their use. ${ }^{8}$ The cost of care among patients treated with AICs has been reported to be lower. ${ }^{4}$ In these data, the patients receiving AICs were compared with patients who did not receive them. The non-AIC groups did not have other standardized measures to prevent infection. Our data address a different question. We compared two protocols designed to reduce infection and saw no additional benefit to the protocol including AICs. To be clear, we did not directly compare AICs and injection of antibiotics into the shunt (which was part of the old protocol). We have described two protocols (or bundles), each including multiple steps. We achieved similar infection rates with both of them. We do not have good evidence that one protocol is better or that any one step in either protocol is sufficient alone.
At present, the choice between our two protocols could be influenced by cost, but we have not done a cost-effectiveness analysis. We have received anecdotal comments from surgeons that the splicing together of the AICs and the valves is inconvenient and an additional source of potential error during the surgery, whereas the old protocol did not impose any restrictions on shunt hardware.

\section{Conclusions}

Compliance with a new standardized protocol reduces the infection rate compared with noncompliance. The overall infection rate is similar to our previous protocol. To inform the development of future protocols, we assessed the impact of the individual steps in the protocol, but our conclusions are based on the impact of the entire protocol (or "bundle"). Further work to reduce shunt infection rates is warranted.

\section{Appendix}

The HCRN currently consists of the following clinical centers and investigators: Primary Children's Hospital, University of Utah (J. Kestle); Children's Hospital of Alabama, University of Alabama at Birmingham (J. Oakes, C. Rozzelle); Hospital for Sick Children, University of Toronto (J. Drake, A. Kulkarni); Texas Children's Hospital, Baylor College of Medicine (T. Luerssen, W. Whitehead); Seattle Children's Hospital, University of Washington (S. Browd, T. Simon); Children's Hospital of Pittsburgh, University of Pittsburgh (M. Tamber); St. Louis Children's Hospital, Washington University in St. Louis (D. Limbrick); Monroe Carell Jr. Children's Hospital at Vanderbilt, Vanderbilt University Medical Center (J. Wellons, R. Naftel, C. Shannon); British Columbia Children's Hospital, University of British Columbia (D. Cochrane); investigator at large (J. RivaCambrin); HCRN Data Coordinating Center, Department of Pediatrics, University of Utah (R. Holubkov).

\section{Acknowledgments}

The HCRN has been funded by National Institute of Neurological Disorders and Stroke (NINDS grant no. 1RC1NS068943-01), Patient Centered Outcome Research Institute (PCORI grant no. CER-1403-13857), The Gerber Foundation (reference no. 16923638), private philanthropy, and the Hydrocephalus Association. We would like to thank our colleagues who kindly agreed to participate in this HCRN project and allow collection of patient data for the purpose of this publication: D. Brockmeyer, M. Walker, R. Bollo, J. Blount, J. Johnston, B. Rocque, L. Ackacpo-Satchivi, P. Dirks, J. Rutka, M. Taylor, D. Curry, R. Dauser, A. Jea, S. Lam, R. Ellenbogen, J. Ojemann, A. Lee, A. Avellino, I. Pollack, S. Greene, E. Tyler-Kabara, T. S. Park, J. Leonard, M. Smyth, N. Tulipan, A. Singhal, and P. Steinbok.

In addition, this work would not have been possible without the outstanding support of the dedicated personnel at each clinical site and the data-coordinating center. Special thanks goes to T. Bach, N. Tattersall (Salt Lake City), A. Arynchyna (Birmingham), H. Ashrafpour (Toronto), S. Ryan (Houston), A. Anderson (Seattle), A. Luther (Pittsburgh), D. Mercer (St. Louis), A. Davis, (Nashville), R. Hengel (British Columbia), and M. Langley and N. Nunn (Utah Data Coordinating Center). We thank them for their hard work and contributions to the HCRN.

\section{References}

1. Berenholtz SM, Pronovost PJ, Lipsett PA, Hobson D, Earsing K, Farley JE, et al: Eliminating catheter-related blood- 
stream infections in the intensive care unit. Crit Care Med 32:2014-2020, 2004

2. Choux M, Genitori L, Lang D, Lena G: Shunt implantation: reducing the incidence of shunt infection. J Neurosurg 77:875-880, 1992

3. Clancy CM: Progress on a national patient safety imperative to eliminate CLABSI. Am J Med Qual 27:170-171, 2012

4. Edwards NC, Engelhart L, Casamento EM, McGirt MJ: Cost-consequence analysis of antibiotic-impregnated shunts and external ventricular drains in hydrocephalus. J Neurosurg 122:139-147, 2015

5. Faillace WJ: A no-touch technique protocol to diminish cerebrospinal fluid shunt infection. Surg Neurol 43:344-350, 1995

6. Hommelstad J, Mads $\varnothing$ A, Eide PK: Significant reduction of shunt infection rate in children below 1 year of age after implementation of a perioperative protocol. Acta Neurochir (Wien) 155:523-531, 2013

7. Kestle JR, Riva-Cambrin J, Wellons JC III, Kulkarni AV, Whitehead WE, Walker ML, et al: A standardized protocol to reduce cerebrospinal fluid shunt infection: the Hydrocephalus Clinical Research Network Quality Improvement Initiative. J Neurosurg Pediatr 8:22-29, 2011

8. Konstantelias AA, Vardakas KZ, Polyzos KA, Tansarli GS, Falagas ME: Antimicrobial-impregnated and -coated shunt catheters for prevention of infections in patients with hydrocephalus: a systematic review and meta-analysis. J Neurosurg 122:1096-1112, 2015

9. Pirotte BJ, Lubansu A, Bruneau M, Loqa C, Van Cutsem N, Brotchi J: Sterile surgical technique for shunt placement reduces the shunt infection rate in children: preliminary analysis of a prospective protocol in 115 consecutive procedures. Childs Nerv Syst 23:1251-1261, 2007

10. Rotim K, Miklic P, Paladino J, Melada A, Marcikic M, Scap M: Reducing the incidence of infection in pediatric cerebrospinal fluid shunt operations. Childs Nerv Syst 13:584-587, 1997

11. Topal J, Conklin S, Camp K, Morris V, Balcezak T, Herbert
P: Prevention of nosocomial catheter-associated urinary tract infections through computerized feedback to physicians and a nurse-directed protocol. Am J Med Qual 20:121-126, 2005

\section{Disclosures}

The authors report no conflict of interest concerning the materials or methods used in this study or the findings specified in this paper. None of the sponsors participated in design and conduct of this study; collection, management, analysis, and interpretation of the data; or preparation, review, or approval of this manuscript. Its contents are solely the responsibility of the authors and do not necessarily represent the official view of the sponsors.

\section{Author Contributions}

Conception and design: Kestle. Acquisition of data: Kestle, Cochrane, Kulkarni, Limbrick, Luerssen, Oakes, Riva-Cambrin, Rozzelle, Simon, Walker, Wellons, Browd, Drake, Shannon, Tamber, Whitehead. Analysis and interpretation of data: Kestle, Holubkov. Drafting the article: Kestle. Critically revising the article: Kestle, Holubkov. Reviewed submitted version of manuscript: all authors. Approved the final version of the manuscript on behalf of all authors: Kestle. Statistical analysis: Kestle, Holubkov. Administrative/technical/material support: Kestle. Study supervision: Kestle.

\section{Supplemental Information \\ Current Affiliations}

Dr. Riva-Cambrin: Department of Clinical Neurosciences, University of Calgary, Alberta, Canada.

\section{Correspondence}

John R. W. Kestle, Division of Pediatric Neurosurgery, 100 N. Mario Capecchi Dr., Ste. 1475, Salt Lake City, UT 84113. email: john.kestle@hsc.utah.edu. 